\title{
PRODUCTS WITH CLOSED PROJECTIONS. II
}

\author{
BY \\ N. NOBLE
}

\begin{abstract}
Conditions under which some or all of the projections on a product space will be closed or $z$-closed are studied, with emphasis on infinite products. These results are applied to characterize normal products up to countably many factors, to characterize closed product maps up to finitely many factors, and to give conditions under which products will be countably compact, Lindelöf, paracompact, $\mathfrak{m}-\mathfrak{n}$ compact, etc. Generalizations of these results to $\mathfrak{n}$-products and box products are also given. Our easily stated results include: All powers of a $T_{1}$ space $X$ are normal if and only if $X$ is compact Hausdorff, all powers of a nontrivial closed map $p$ are closed if and only if $p$ is proper, the product of countably many Lindelöf $P$-spaces is Lindelöf; and the product of countably many countably compact sequential spaces is countably compact sequential.
\end{abstract}

Introduction. All hypothesized spaces are assumed to not be indiscrete. In [19] conditions under which one or both of the projections on a product space $X \times Y$ would be closed (map closed sets to closed sets) or $z$-closed (map zero sets to closed sets) were investigated, and various applications were given. Closed and $z$-closed projections have also been investigated and/or applied in [5], [12], [3], [26], [28], [29], [20], [21], [22] and [30], always for finite products. In this paper we consider conditions under which infinite products will have some or all of their projections closed or $z$-closed $(\S 1)$ and give applications to normal products $(\S 2)$, products of closed maps $(\S 3)$, and products satisfying various compactness properties (\$4). (By projections on a product space we mean the natural maps from the product onto subproducts, not just those onto factors.) In the final section, we note some generalizations of our results to certain nonstandard products $(\mathfrak{n}$ products and box products).

1. Projections on infinite products. We begin with the description of those products each of whose projections is $z$-closed. Recall that a space $X$ is pseudocompact if each continuous real-valued function on $X$ is bounded, and a $P$-space if each $G_{\delta}$ in $X$ is open. For $X=\prod_{\alpha \in A} X_{\alpha}$, the subproduct $\prod_{\alpha \in B} X_{\alpha}$ will be denoted as $X_{B}$ and the projection from $X$ to $X_{B}$ will be denoted as $\pi_{B}$. Of course, $X_{\{\alpha\}}$ and $\pi_{\{\alpha\}}$ will be denoted as $X_{\alpha}$ and $\pi_{\alpha}$.

1.1. TheOREM. Let $X=\prod_{\alpha \in A} X_{\alpha}$ be completely regular with $A$ infinite. Each projection on $X$ is $z$-closed if and only if $X$ is pseudocompact.

Received by the editors February 4, 1970.

AMS 1968 subject classifications. Primary 5423, 5425, 5440, 5450.

Key words and phrases. Closed projections, $z$-closed projections, normal products, paracompact products, Lindelöf products, countably compact products, $\mathfrak{m}$-n-compact products, $\mathfrak{n}$-products, box products, products of closed maps.

Copyright (C) 1971, American Mathematical Society 
Proof. If $X$ is pseudocompact, each projection is $z$-closed by [30, Theorem 1] or [19, Theorem 3.4]. For the converse, write $A=B \cup C$ with $B$ and $C$ disjoint and infinite, and note that $X_{B}$ and $X_{C}$ cannot be $P$-spaces (since, by our standing hypothesis, no $X_{\alpha}$ is indiscrete and the product of infinitely many nonindiscrete spaces is never a $P$-space). Since the projections on $X=X_{B} \times X_{C}$ are $z$-closed, it follows by [19, Theorem 3.4] that $X$ is pseudocompact.

It is shown in [9] that a product space is pseudocompact if and only if each countable subproduct is pseudocompact, so to show that the projections are $z$-closed only countable subproducts need be considered. For some conditions under which infinite products will be pseudocompact, see [23].

Let $\mathfrak{m}$ and $\mathfrak{n}$ be infinite cardinals. A space is $\mathfrak{m}-\mathfrak{n}$-compact if each $\mathfrak{m}$-fold open cover has a subcover of cardinality less than $\mathfrak{n}$, and a space is $\mathfrak{n}$-compact if it $\mathfrak{m}$-n-compact for each $\mathfrak{m}$. Thus $\boldsymbol{\aleph}_{0}$-compact $=$ compact, $\boldsymbol{\aleph}_{1}$-compact $=$ Lindelöf, and $\boldsymbol{\aleph}_{0}-\boldsymbol{\aleph}_{0}$-compact $=$ countably compact. Our next result will follow as a special case of Theorem 1.6.

1.2. Theorem. Let $X=\prod_{\alpha \in A} X_{\alpha}$ where card $A=\mathfrak{n}$. Each projection on $X$ is closed if and only if $X$ is $\mathfrak{n}-\aleph_{0}$-compact and, for each finite $F \subseteq A$, each projection on $X_{F}$ is closed.

It follows that all powers of a space $X$ have all of their projections closed if and only if $X$ is compact. (The projection along a compact space is always closed.) For a cardinal $\mathfrak{n}$ we adopt the following conventions: We identify $\mathfrak{n}$ with the smallest ordinal of cardinality $\mathfrak{n}$, so $\mathfrak{n}$ is the ordered set of all ordinals of cardinality less than $\mathfrak{n}$. When considered as a topological space, $\mathfrak{n}$ (the ordinal) is assumed to have the usual (interval) topology. Following [18] we call a net indexed by (i.e., with domain) $D$ a $D$-net. Thus by our convention an $\mathfrak{n}$-net is a net indexed by the ordinal $\mathfrak{n}$. Let $D(\mathfrak{m}, \mathfrak{n})$ denote the set of subsets $S$ of $\mathfrak{m}$ with card $S<\mathfrak{n}$, ordered by inclusion. We call a net an $\mathfrak{m}$ - $\mathfrak{n}$-sequence if, for some $\mathfrak{m}^{\prime}$ satisfying $\mathfrak{n} \leqq \mathfrak{m}^{\prime} \leqq \mathfrak{m}$, it is a $D\left(\mathfrak{m}^{\prime}, \mathfrak{n}\right)$-net. In [30], [18] and [11] $\mathfrak{n}-\aleph_{0}$-sequences are called, respectively, $\mathfrak{n}$-phalanxes, $\mathfrak{n}$-nets and $\mathfrak{n}$-sequences. Clearly as instruments of convergence, $\aleph_{0}-\aleph_{0}$-sequences are equivalent to sequences. Indeed, for any regular cardinal $\mathfrak{n}$, $\mathfrak{n}$-n-sequences are equivalent, in this way, to $\mathfrak{n}$-nets. Our next result follows by a straightforward adaptation of the proof of [18, Lemma 2] which is the case $\mathfrak{n}=\boldsymbol{\aleph}_{0}$.

1.3. Proposition. A space $X$ is $\mathfrak{m}-\mathfrak{n}$-compact if and only if each $\mathfrak{m}-\mathfrak{n}$-sequence in $X$ has a cluster point.

Incidentally, the same proof can be adapted to show that a space $X$ is $\mathfrak{n}$-ncompact if and only if each $\mathfrak{n}$-net in $X$ has a cluster point. (For $\mathfrak{n}=\boldsymbol{\aleph}_{0}$ this is the familiar result: $X$ is countably compact if and only if each countable subset has an accumulation point.) Recall that a space is called $\mathfrak{n}$-discrete if each $\mathfrak{n}$-fold intersection of open sets is open, and $<\mathfrak{n}$-discrete if it is $\mathfrak{m}$-discrete for each $\mathfrak{m}<\mathfrak{n}$. Thus $\boldsymbol{N}_{0}$-discrete $=$ pseudo-discrete $=P$-space. The $D$-nets of a space are said to 
determine its topology if a subset is closed whenever it contains the limits of each of its convergent $D$-nets (i.e., if it has the weak topology with respect to its “convergent $D$-nets" where "convergent $D$-nets" are assumed to contain their limit points). A space whose topology is determined by its $\mathfrak{m}$-n-sequences will be called an m-n-sequential space. Thus $\aleph_{0}-\aleph_{0}$-sequential=sequential, and $\mathfrak{m}-\aleph_{0}$-sequential $=$ the $\mathfrak{m}$-sequential of [11]. Note that if $X$ is $<\mathfrak{n}$-discrete and each point of $X$ has a neighborhood basis of cardinality at most $m$, then each point has an $\mathfrak{m}$-n-sequence of neighborhoods $\left\{U_{\lambda}: \lambda \in D(\mathfrak{m}, \mathfrak{n})\right\}$, with $U_{\lambda} \supseteq U_{\gamma}$ for $\lambda \leqq \gamma$, which forms a basis for $x$. (Choose any basis indexed by $\mathfrak{m}$ and adjoin all $<\mathfrak{n}$-fold intersections.) Thus $X$ is $\mathfrak{m}$-n-sequential.

1.4. THEOREM. The following conditions on $X$ are equivalent:

(i) For each $\mathrm{m}-\mathfrak{n}$-sequential space $Y, \pi: X \times Y \rightarrow Y$ is closed.

(ii) For each subspace $Y$ of an $\mathfrak{m}$-n-sequential space, $\pi: X \times Y \rightarrow Y$ is closed.

(iii) $X$ is $\mathfrak{m}$-n-compact.

Proof. (i) =(ii). If $\pi: X \times Y \rightarrow Y$ is closed and $Y^{\prime} \subseteq Y$, then the restriction of $\pi$ to $X \times Y^{\prime}$ is closed.

(ii) $=$ (iii). By [19, Theorem 2.2], a space satisfying (ii) must be $\mathfrak{n}^{\prime}$ - $\mathfrak{n}^{\prime}$-compact for each $\mathfrak{n}^{\prime}$ between $\mathfrak{m}$ and $\mathfrak{n}$.

(iii) =(i). This follows by Proposition 1.3 and the Theorem of [5] (or by the obvious direct proof).

For a parallel result for $z$-closed projections, see [12, Proposition 5.3]. We will give applications of Theorem 1.4 in $\$ 4$.

1.5. Lemma. Let $X=\prod_{\alpha \in A} X_{\alpha}$ with card $(A)=\mathfrak{n}$, and let $H \subseteq X$ be $\mathfrak{n}-\aleph_{0}$-compact. If $U \supseteq H$ is open, then there exists a finite $F \subseteq A$ and an open $V \supseteq \pi_{F} H$ such that $V \times X_{A \mid F} \subseteq U$.

Proof. For each finite subset $B$ of $A$, let $U_{B}$ be the interior of

$$
\left\{x \in X_{B}:\{x\} \times X_{A \mid B} \subseteq U\right\} .
$$

For each $y$ in $H$ there exists a basic open neighborhood $\prod_{\alpha \in C} U_{\alpha} \times X_{A \mid C} \leqq U$ with $C$ finite, so $y$ is in $U_{C}$. Thus $\left\{U_{B}: B \subseteq A\right.$ is finite $\}$ is an $\mathfrak{n}$-fold open cover of $H$, so there exists a finite subcovering $\left\{U_{B_{i}}: 1 \leqq i \leqq n\right\}$. Then $F=\bigcup_{i=1}^{n} B_{i}$ and $V=\pi_{C}\left(\bigcup_{i=1}^{n} \pi_{B_{i}}^{-1}\left(U_{B_{i}}\right)\right)$ have the desired properties.

1.6. TheOREM. Let $X=\prod_{\alpha \in A} X_{\alpha}$ where card $A=\mathfrak{n}$ and let $H=\prod_{\alpha \in A} H_{\alpha}$ where each $H_{\alpha} \subseteq X_{\alpha}$. Each projection $\prod_{\alpha \in B} H_{\alpha} \times X_{A \backslash B} \rightarrow X_{A \backslash B}$ is closed if and only if $H$ is $\mathrm{n}-\aleph_{0}$-compact and for each finite $F \subseteq A$, and for each $B \subseteq F$, the projection $\prod_{\alpha \in B} H_{\alpha} \times X_{F \backslash B} \rightarrow X_{F}$ is closed.

Proof. First suppose each projection $\prod_{\alpha \in B} H_{\alpha} \times X_{A \backslash B} \rightarrow X_{A \backslash B}$ is closed; it suffices to show that $H$ is $\mathfrak{n}-\aleph_{0}$-compact. Write $A=B \cup C$ where $B$ and $C$ are disjoint, each of cardinality $\mathfrak{n}$, and set $H^{\prime}=\prod_{\alpha \in B} H_{\alpha}, H^{\prime \prime}=\prod_{\alpha^{\prime} C} H_{\alpha}$. Note that for each infinite cardinal $\mathfrak{m} \leqq \mathfrak{n}, X_{B}$ contains a point $x$ and a collection of closed sets 
$\left\{S_{\lambda}: \lambda \in \mathfrak{m}\right\}$ such that $x$ is in the closure of $\bigcup_{\lambda \in \mathfrak{m}} S_{\lambda}$ but is not in the closure of $\bigcup_{\lambda<\lambda_{0}} S_{\lambda}$ for any $\lambda_{0}$ in $\mathfrak{m}$ (indeed, assuming without loss of generality that $\mathfrak{m} \subseteq B$, we may take $S_{\lambda}$ to be a point whose $\alpha$ th coordinate coincides with that of $x$ except for those $\alpha \in \mathfrak{m}$ satisfying $\alpha>\lambda$ ). Since the projection $H^{\prime \prime} \times X_{B} \rightarrow X_{B}$ is closed, it follows by [19, Theorem 2.2] that $H^{\prime \prime}$ is $\mathfrak{m}$-m-compact. Since this is true for each infinite $\mathfrak{m} \leqq \mathfrak{n}, H^{\prime \prime}$ is $\mathfrak{n}-\aleph_{0}$-compact. Similarly, $H^{\prime}$ is $\mathfrak{n}-\aleph_{0}$-compact, so $\pi: H^{\prime} \times H^{\prime \prime} \rightarrow H^{\prime \prime}$ is a closed continuous map with $\mathfrak{n}-\aleph_{0}$-compact range and each fiber $\mathfrak{n}-\aleph_{0}$-compact. It follows by [6, Proposition 1.1] that $H=H^{\prime} \times H^{\prime \prime}$ is $\mathfrak{n}-\aleph_{0}$-compact.

Now suppose that $H$ is $\mathfrak{n}-\aleph_{0}$-compact and that for each finite $F \subseteq A$ and each $B \subseteq F$, the projection $\prod_{\alpha \in B} H_{\alpha} \times X_{F \backslash B} \rightarrow X_{F \backslash B}$ is closed. We first show that for $F \subseteq A$ finite the projection $\pi: \prod_{\alpha \in A \backslash F} H_{\alpha} \times X_{F} \rightarrow X_{F}$ is closed. Let $S \subseteq \prod_{\alpha \in A \backslash F} H_{\alpha} \times X_{F}$ be a closed set and let $x$ be any point in the closure of $\pi S$. Let $B \subseteq A$ be finite with $B \cap F=\varnothing$; since the projection $\prod_{\alpha \in B} H_{\alpha} \times X_{F} \rightarrow X_{F}$ is closed, there exists a point $x_{B}$ in $\prod_{\alpha \in B} H_{\alpha}$ such that $\left(x_{B}, x\right)$ is in the closure of the projection of $S$ onto $\prod_{\alpha \in B} H_{\alpha} \times X_{F}$. For each such $B$, choose $x_{B}^{\prime}$ in $\prod_{\alpha \in A \backslash F} H_{\alpha}$ such that the $\alpha$ th coordinants of $x_{B}$ and $x_{B}^{\prime}$ coincide for each $\alpha$ in $B$. Since $\prod_{\alpha \in A \mid F} H_{\alpha}$ is $\mathfrak{n}-\aleph_{0}$-compact, the $\mathfrak{n}-\aleph_{0}$-sequence $\left\{x_{B}^{\prime}: B \subseteq A \backslash F\right.$ is finite $\}$ has a cluster point, $y$. But $(y, x)$ is clearly in the closure of $S$, so $x$ is in $\pi S$. Therefore $\pi S$ is closed.

Finally, let $B \subseteq A$ be arbitrary, let $S \subseteq \prod_{\alpha \in B} H_{\alpha} \times X_{A \backslash B}$ be closed, and suppose $x$ is in $X_{A \backslash B} \mid \pi S$. Since $\pi^{-1}(x)$ is $\mathfrak{n}-\aleph_{0}$-compact, there exists a finite $F \subseteq A$ such that $\left(\pi_{F}\left(\pi^{-1}(x)\right) \times X_{A \mid F}\right) \cap S=\varnothing$ (by Lemma 1.5). Set $C=F \cap(A \backslash B)$; clearly $C \neq \varnothing$. Also, $\pi_{C}(x)$ is not in the closed set $\pi_{C} S$, so for $U=X_{C} \mid \pi_{C} S, U \times X_{A \mid(B \cup C)}$ is a neighborhood of $x$ which does not meet $\pi S$. Thus $x$ is not in the closure of $\pi S$ so $\pi S$ is closed. Therefore $\pi$ is closed.

1.7. EXAMPLE. For each infinite cardinal $\mathfrak{n}$ there exists a space $Y$ such that each projection on $Y^{\mathfrak{n}}$ is closed, but for $\mathfrak{m}>\mathfrak{n} Y^{\mathfrak{m}}$ has projections which are not closed.

Proof. Let $Y$ be the successor (as a cardinal) of $\mathfrak{n}$ and note that each subset of $Y$ of cardinality $\mathfrak{n}$ has compact closure. Since this property is preserved by products, all powers of $Y$ are $\mathfrak{n}-\aleph_{0}$-compact (using Proposition 1.3). Since each point of $Y$ has a base of cardinality at most $\mathfrak{n}, Y^{\mathfrak{m}}$ also has this property for $\mathfrak{m} \leqq \mathfrak{n}$ so by Theorem 1.4 all projections on $Y^{\mathfrak{n}}$ are closed. Since $Y$ is not compact, $Y$ and hence $Y^{Y}$ are not $Y-\aleph_{0}$-compact so by Theorem 1.2 not all the projections on $Y^{Y}$ are closed.

Note that $Y=N$, the natural numbers, is an example of a space such that $Y^{n}$ has all its projections closed for each integer $n$ while $Y^{N}$ does not. The results above, and those to come, raise the question: Under what conditions are $\mathfrak{n}$-fold products $\mathfrak{n}-\boldsymbol{\aleph}_{0}$-compact? We will consider this question in $\$ 4$; our results make use of the result below.

1.8. TheOREM. Let $X=\prod_{\alpha \in \mathfrak{n}} X_{\alpha}$, where $\mathfrak{n}$ is infinite. If for each $\alpha_{0}$ in $\mathfrak{n}$ the projection $\prod_{\alpha \leqq \alpha_{0}} X_{\alpha} \rightarrow \prod_{\alpha<\alpha_{0}} X_{\alpha}$ is closed, then for each such $\alpha_{0}$ the projection $\pi^{\alpha}{ }_{0}: X \rightarrow \prod_{\alpha<\alpha_{0}} X_{\alpha}$ is also closed. 
Proof. Let $S \leqq X$ be such that $\pi^{\alpha} 0(S)$ is not closed; we show that $S$ is not closed. Choose a point $x^{0}$ in cl $\left(\pi^{\alpha} 0 S\right) \mid \pi^{\alpha} 0 S$ and for $\alpha<\alpha_{0}$ let $x_{\alpha}$ be the $\alpha$ th coordinant of $x^{0}$. Let $\beta$ be greater than or equal to $\alpha_{0}$ and suppose inductively that for each $\alpha<\beta, x_{\alpha}$ has been chosen so that for $x^{\beta}$ the point $\left(x_{\alpha}\right)$ in $\prod_{\alpha<\beta} X_{\alpha}, x^{\beta}$ is in the closure of $\pi^{\beta}(S)$. Since for $\pi: \prod_{\alpha \leqq \beta} X_{\alpha} \rightarrow \prod_{\alpha<\beta} X_{\alpha}$ the projection $\pi\left(\mathrm{cl} \pi^{\beta+1}(S)\right)$ is a set which contains $\pi^{\beta}(S)$, there exists a point $x_{\beta}$ in $X_{\beta}$ such that $\left(x_{\beta}, x^{\beta}\right)$ is in the closure of $\pi^{\beta+1}(S)$. Thus we construct, inductively, a point $x$ in $X$ which is not in $S$ (since $\pi^{\alpha} 0(x)=x^{0}$ is not in $S$ ) such that for each $\beta$ in $\mathfrak{n}, \pi^{\beta}(x)$ is in the closure of $\pi^{\beta}(S)$. Clearly $x$ must be in the closure of $S$, so $S$ is not closed.

The analogue of Theorem 1.8 for $z$-closed projections fails: If it held then (as in the proof of Theorem 4.1) one could show that a product of pseudocompact spaces is pseudocompact whenever each finite subproduct is. That this is false is shown by examples in [2], [7] and [8].

\section{Normal products.}

2.1. Theorem. Let $X=\prod_{\alpha \in A} X_{\alpha}$ where card $A=\mathfrak{n}$ and suppose each finite subproduct of $X$ is normal $T_{1}$.

(i) If $X$ is $\mathfrak{n}-\aleph_{0}$-compact then $X$ is normal.

(ii) If $X$ is normal then some product of all but countably many of the factors is $\mathfrak{n}-\aleph_{0}$-compact.

Proof. (i) By [12, Proposition 5.5] a projection on a normal $T_{1}$ space is closed if and only if it is $z$-closed. Since each finite subproduct of $X$ is normal $T_{1}$ and pseudocompact, each finite subproduct has all of its projections closed, so by Theorem 1.2 each projection on $X$ is closed.

Let $H$ and $H^{\prime}$ be disjoint closed subsets of $X$; since $H$ is $\mathfrak{n}$ - $\aleph_{0}$-compact, there exists a finite $F \subseteq A$ such that $\left(\pi_{F} H \times X_{A \mid F}\right) \cap H^{\prime}=\varnothing$ (by Lemma 1.5). But then $\pi_{F} H$ and $\pi_{F} H^{\prime}$ are disjoint closed subsets of the normal space $X_{F}$, so they have disjoint neighborhoods $U$ and $V$ respectively. Then $\pi_{F}^{-1}(U)$ and $\pi_{F}^{-1}(V)$ are disjoint neighborhoods of $H$ and $H^{\prime}$, as desired.

(ii) Since $X$ is normal $T_{1}$, there exists a countable subset $B \subseteq A$ such that $X_{A \backslash B}$ is countable compact, hence pseudocompact (by the second Theorem of [24]). By Theorem 1.1, each projection on $X_{A \backslash B}$ is $z$-closed, so by the result quoted above each projection on $X_{A \backslash B}$ is in fact closed. By Theorem 1.2, $X_{A \backslash B}$ is $\mathfrak{n}-\aleph_{0}$-compact.

2.2. Corollary. All powers of a $T_{1}$-space $Y$ are normal if and only if $Y$ is compact $T_{2}$.

2.3. Corollary. A c-fold product of separable $T_{1}$ spaces is normal only if all but countably many of the factors are compact. Thus assuming the continuum hypothesis, the product of uncountably many copies of a separable $T_{1}$ space $Y$ is normal if and only if $Y$ is compact $T_{2}$. 
Proof. For $\mathfrak{n}$ the successor of $c$, each completely regular separable space is $\mathfrak{n}$ compact-indeed it has a basis of cardinality $c$, the cozero sets. (Since each continuous real-valued function is determined by its values on the countable dense subset, there are only $c^{\aleph_{0}}=c$ such functions and hence only $c$ cozero sets.)

It follows of course that if a product $\prod_{\alpha \in A} X_{\alpha}$ is normal where card $A=c$, then for all but countably many $\alpha$ each countable subset of $X_{\alpha}$ has compact closure. By a similar argument it follows that if $X^{\mathfrak{m}}$ is normal for $\mathfrak{m} \geqq 2^{\mathfrak{n}}$, then each $\mathfrak{n}$-fold subset of $X$ has compact closure. In the converse direction, see Example 2.7.

Since countable products of, for instance, metric spaces are normal without any of the factors being countably compact, part (ii) of 2.1 cannot be improved in the obvious way. It can be improved in nonobvious ways-for example, Morita shows in [17, Theorem 2.4] that for $I$ the unit interval, $Y \times I^{\mathfrak{n}}$ is normal if and only if $Y$ is $\mathfrak{n}$-paracompact and normal (a space is $\mathfrak{n}$-paracompact if each $\mathfrak{n}$-fold open cover admits an open locally finite refinement). Thus if $X=\prod_{\alpha \in A} X_{\alpha}$ is normal and, for $\mathfrak{n}>\boldsymbol{\aleph}_{0}, \mathfrak{n}$-many of the $X_{\alpha}$ contain a copy of $I$, the product $Y$ of the countably many factors not treated by (ii) must be $\mathfrak{n}$-paracompact. (And if $Y$ is countably compact normal, it will therefore be $\mathfrak{n}-\aleph_{0}$-compact by [17, Theorem 1.8].) At any rate, Theorem 2.1 raises the question of when the product of two spaces, one of which is countably compact, will be normal. Our next result will give some information on this. We call a collection of open subsets of a space $Y$ a hyperbasis for $Y$ if for each closed subset $F$ of $Y$ and each open $U \supseteq F$, there exists a member $V$ of the collection with $F \subseteq V \subseteq U$. I.e., a hyperbasis is a basis for the neighborhoods of the closed sets. The theorem below follows by a straightforward adaptation of the proof of [19, Theorem 2.2].

2.4. TheOREM. Let $X$ and $Y$ be normal with $\pi: X \times Y \rightarrow Y$ closed. If, for some cardinal $\mathfrak{n}, Y$ is $\mathfrak{n}$-paracompact and $X$ has a hyperbasis of cardinality $\mathfrak{n}$, then $X \times Y$ is normal.

To apply Theorem 2.4 , note that if $X$ is $\mathfrak{n}$-compact and $<\mathfrak{n}$-discrete, each basis of $X$ which is closed under $<\mathfrak{n}$-fold unions is a hyperbasis, and if $X$ is normal and contains a dense subset of cardinality $\mathfrak{n}$, the cozero sets of $X$ form a hyperbasis of cardinality at most $c^{\mathfrak{n}}$. (For sharper bounds on the cardinality of the family of cozero sets, see [4].)

2.5. COROllary. If $X$ is $\mathfrak{n}-\aleph_{0}$-compact normal and $Y$ is any paracompact space which can be embedded as a subspace of an $\mathfrak{n}-\aleph_{0}$-sequential space, then $X \times Y$ is normal.

This corollary is a slight improvement of [17, Theorem 4.1]. That the product of a normal space with a sequential, indeed metrizable, space need not be normal is shown by Michael in [15].

2.6. Corollary. Let $X$ and $Y$ be normal $\mathfrak{n}$-paracompact and suppose that $X$ has a hyperbasis of cardinality $\mathfrak{n}$ and $\pi: X \times Y \rightarrow Y$ is closed. Then $X \times Y$ is $\mathfrak{n}$-paracompact (and normal). 
Proof. By Morita's characterization of $\mathfrak{n}$-paracompactness, we need only show that $Y \times X \times I^{\mathfrak{n}}$ is normal; but since clearly $X \times I^{\mathfrak{n}}$ has a hyperbasis of cardinality $\mathfrak{n}$, and $\pi: X \times I^{\mathfrak{n}} \times Y \rightarrow Y$ is closed, this follows by Theorem 2.4.

If $X$ is collectionwise normal, then the hypothesis of Theorem 2.4 guarantees that $X \times Y$ will be collectionwise normal-this is shown by the proof of [17, Corollary 2.3]. We close this section with an example showing that arbitrarily large products can be normal without having compact factors.

2.7. EXAMPLE. For each infinite cardinal $\mathfrak{n}$ there exists a space $Y$ such that $Y^{\mathfrak{n}}$ is normal but $Y^{\mathfrak{m}}$ is not normal for $\mathfrak{m}>\mathfrak{n}$.

Proof. Let $Y$ be as in Example 1.7. By that example and Theorem 2.1, we need only show that $Y^{n}$ is normal for each integer $n$. The space $Y$ is normal. Suppose inductively that $Y^{n-1}$ is normal and let $H$ and $H^{\prime}$ be disjoint closed subsets of $\prod_{i=1}^{n} Y_{i}$, where each $Y_{i}$ is a copy of $Y$. Then there exists an index $j$ and a $\lambda_{0}$ in $Y$ such that, for $Y^{*}$ the set of elements of $Y_{j}$ less than or equal to $\lambda_{0}$, either $H$ or $H^{\prime}$ is contained in $Y^{*} \times \prod\left\{Y_{i}: 1 \leqq i \leqq n, i \neq j\right\}$; otherwise there would exist a sequence $y_{n}$ increasing in each coordinate with $y_{n}$ in $H$ for $n$ even and $y_{n}$ in $H^{\prime}$ for $n$ odd, and the coordinatewise supremum of the $y_{n}$ would be a point in $H \cap H^{\prime}$. Since $Y^{*}$ is open and closed in $Y_{j}$, it suffices to show that $Y^{*} \times Y^{n-1}$ is normal. Since the projection $Y^{n-1} \times Y^{*} \rightarrow Y^{*}$ is closed and $Y^{*}$ (being compact) is paracompact, this follows by Theorem 2.4 .

3. Products of closed maps. In this section, "map" will mean "continuous onto function". By our standing hypothesis the range of an hypothesized map cannot be indiscrete, so let us note that the class of closed maps whose ranges are indiscrete is closed under arbitrary products, and furthermore the product of such a map with a closed map is always closed. Recall that a fiber of a map $p$ is a set of the form $p^{-1}(y)$ for $y$ a point in the range of $p$. A subset of a product $\prod_{\alpha \in A} X_{\alpha}$ is called a product set (with respect to $\prod_{\alpha \in A} X_{\alpha}$ ) if it has the form $\prod_{\alpha \in A} F_{\alpha}$ where for each $\alpha, F_{\alpha} \subseteq X_{\alpha}$. A neighborhood of a set $F \subseteq X$ is a subset of $X$ whose interior contains $F$.

3.1. Proposition. Let $\left\{p_{\alpha}: X_{\alpha} \rightarrow Y_{\alpha} \mid \alpha \in A\right\}$ be a collection of closed maps. The map $p=\prod_{\alpha \in A} p_{\alpha}$ is closed if and only if for each choice of fibers $F_{\alpha}$ of $p_{\alpha}$, every neighborhood of $\prod_{\alpha \in A} F_{\alpha}$ contains a product set neighborhood.

Proof. Suppose $p$ is closed, choose fibers $F_{\alpha}$ of $p_{\alpha}$ and let $U$ be any open neighborhood of $F=\prod_{\alpha \in A} F_{\alpha}$. For $H$ the complement of $U, p(H)$ is closed and does not contain the point $p(F)$ so there exists an open neighborhood $\prod_{\alpha \in A} V_{\alpha}$ of $p(F)$ which does not meet $p(H)$. (Where each $V_{\alpha}$ is open and $V_{\alpha}=X_{\alpha}$ with at most finitely many exceptions.) The set $\prod_{\alpha \in A} p_{\alpha}^{-1}\left(V_{\alpha}\right)$ is a product set neighborhood of $F$ which is contained in $U$.

Now suppose that each fiber of $p$ has a basis of product set neighborhoods, let $H \subseteq \prod_{\alpha \in A} X_{\alpha}$ be closed and let $y$ be any point in the complement of $p(H)$. Since the 
complement of $H$ is a neighborhood of $p^{-1}(y)$, it contains an open product set neighborhood $U=\prod_{\alpha \in B} U_{\alpha} \times \prod_{\alpha \in A \backslash B} X_{\alpha}$. Since the complement, $H^{\prime}$, of $U$ is $\bigcup_{\alpha \in B} \pi_{\alpha}^{-1}\left(X_{\alpha} \mid U_{\alpha}\right)$ it is clear that $p\left(H^{\prime}\right)$ is closed. Since $p(H) \subseteq p\left(H^{\prime}\right)$ and $y$ is not in $p\left(H^{\prime}\right)$, it follows that $y$ is not in the closure of $p(H)$. Thus $p(H)$ is closed, so $p$ is closed.

3.2. TheOREM. Let $p=\prod_{\alpha \in A} p_{\alpha}$ where card $A=\mathfrak{n}$. The map $p$ is closed if and only if each finite subproduct is closed and for each choice of fibers $F_{\alpha}$ of $p_{\alpha}, \prod_{\alpha \in A} F_{\alpha}$ is $\mathfrak{n}$-א $\aleph_{0}$-compact.

Proof. First suppose each finite subproduct is closed and each product of fibers is $\mathfrak{n}-\aleph_{0}$-compact. Let $F=\prod_{\alpha \in A} F_{\alpha}$ be a product of fibers and let $U \supseteq F$ be open in $X=\prod_{\alpha \in A} X_{\alpha}$, the product of the domains of the $p_{\alpha}$. By Proposition 3.1 it suffices to show that $U$ contains a product set neighborhood. But by Lemma 1.5 there exists a finite $B \subseteq A$ and an open $V \subseteq X_{B}$ such that $F \subseteq V \times X_{A \mid B} \subseteq U$ and by the hypothesis (and 3.1) there exists a product set neighborhood $W$ with $\prod_{\alpha \in B} F_{\alpha} \subseteq W$ $\subseteq V$. But then $W \times X_{A \backslash B}$ is as desired.

Now suppose $p$ is closed. Clearly each finite subproduct of $p$ is closed. That each fiber $\prod_{\alpha \in A} F_{\alpha}$ of $p$ is $\mathfrak{n}-\aleph_{0}$-compact will follow from Corollary 3.6.

3.3. TheOREM. Let $p: X \rightarrow X^{\prime}$ and $q: Y \rightarrow Y^{\prime}$ be closed maps. If for each pair of fibers $F$ of $p$ and $F^{\prime}$ of $q$ the projections from $F \times Y$ to $Y$ and from $X \times F^{\prime}$ to $X$ are closed, then $p \times q$ is closed.

Proof. We will apply Proposition 3.1, so let $W$ be an open neighborhood of $F \times F^{\prime}$. For $x$ in $F$ choose, for each $y$ in $F^{\prime}$, neighborhoods $U_{y}$ of $x$ and $V_{y}$ of $y$ such that $U_{y} \times V_{y} \subseteq W$. Since $\pi: X \times F^{\prime} \rightarrow X$ is closed, there exists a neighborhood $U^{x}$ of $x$ such that $U^{x} \subseteq \bigcap\left\{U_{y}: y \in F^{\prime}\right\}$. Set $V^{x}=\bigcup\left\{V_{y}: u \in F^{\prime}\right\}$ and note that $\{x\} \times F^{\prime} \subseteq U^{x} \times V^{x} \subseteq W$. Since $\pi: F \times Y \rightarrow Y$ is closed, there exists a neighborhood $V$ of $F^{\prime}$ such that $V \subseteq \bigcap\left\{V^{x}: x \in F\right\}$. But for $U=\bigcup\left\{U^{x}: x \in F\right\}, F \times F^{\prime} \subseteq U \times V$ $\subseteq W$ as desired.

3.4. THEOREM. The map $p \times 1_{Y}$ is closed if and only if for each fiber $F$ of $p$ the projection $F \times Y \rightarrow Y$ is closed.

Proof. That $p \times 1_{Y}$ is closed when the projection along each fiber is closed follows from Theorem 3.3, so suppose $p \times 1_{Y}$ is closed. Then for any fiber $F$ the restriction of $p \times 1_{Y}$ to $F \times Y$ is closed; but this restriction is just the projection.

3.5. Corollary. (i) $p \times 1_{Y}$ is closed for each closed map $p$ if and only if $Y$ is discrete.

(ii) $p \times 1_{Y}$ is closed for each closed map $p$ each fiber of which is Lindelöf if and only if $Y$ is a $P$-space.

(iii) $p \times 1_{Y}$ is closed for each closed map each fiber of which is countably compact (i.e., for each quasi-proper map $p$ ) if $Y$ is sequential (or a subspace of a sequential space). 
(iv) $p \times 1_{Y}$ is closed for each sequential space $Y$ if and only if $p$ is closed and each fiber of $p$ is countably compact.

(v) $p \times 1_{Y}$ is closed for each $P$-space $Y$ if and only if $p$ is closed and each fiber of $p$ is Lindelöf.

(vi) $p \times 1_{Y}$ is closed for each $Y$ if and only if $p$ is proper.

(vii) $p \times q$ is closed for each closed map $q$ if and only if $p$ is proper and the range of $p$ is discrete.

Proof. The necessary information about closed projections appears in [19].

Part (vi) is of course known. Indeed, it is Bourbaki's definition of a proper map. For $F \subseteq X$, let $X / F$ denote the quotient space formed by identifying $F$ to a point and let $p_{F}$ denote the corresponding quotient map. Note that $p_{F}$ is closed when $F$ is closed. (And almost conversely; if $p_{F}$ is closed and $x$ is in the closure of $F$ but is not in $F$, then $x$ must be in the closure of each point of $F$.)

3.6. Corollary. Let $F=\prod_{\alpha \in A} F_{\alpha}$ where each $F_{\alpha} \subseteq X_{\alpha}$ is closed and card $A=\mathfrak{n}$. If each neighborhood of $F$ contains a product set neighborhood, then for each $B \subseteq A$ the projection $\prod_{\alpha \in B} F_{\alpha} \times \prod_{\alpha \in A \mid B} X_{\alpha} / F_{\alpha} \rightarrow \prod_{\alpha \in A \mid B} X_{\alpha} / F_{\alpha}$ is closed. Thus $F$ is $\mathfrak{n}-\aleph_{0}-$ compact.

Proof. Clearly each subproduct of $F$ also has the property that each neighborhood contains a product neighborhood, so the map $p=\prod_{\alpha \in A} p_{F_{\alpha}}$ is closed (by Proposition 3.1). For $B \subseteq A, p=\left(1_{X_{B}} \times \prod_{\alpha \in A \backslash B} p_{F_{\alpha}}\right) \circ\left(\prod_{\alpha \in B} p_{F_{\alpha}} \times 1_{Y}\right)$ for $Y=\prod_{\alpha \in A \backslash B} X / F_{\alpha}$ so the last factor is closed and hence, by Theorem 3.4, the projection $\prod_{\alpha \in B} F_{\alpha} \times \prod_{\alpha \in A \mid B} X_{\alpha} / F_{\alpha} \rightarrow \prod_{\alpha \in A \mid B} X_{\alpha} / F_{\alpha}$ is closed. It follows as in the proof of Theorem 1.2 that $F$ must be $\mathfrak{n}-\aleph_{0}$-compact.

Recall that Wallace's Theorem [13] states that $F \times F^{\prime}$ has a basis of product set neighborhoods if $F$ and $F^{\prime}$ are compact. Corollary 3.6, and the special cases of Theorems 3.2, 3.3 and 3.4 where each $p_{\alpha}$ has the form $p_{F_{u}}$, may be thought of as describing conditions under which generalizations of Wallace's Theorem will hold. Thus Theorem 3.2 reduces the problem (when does $F=\prod_{\alpha \in A} F_{\alpha}$ have a basis of product set neighborhoods) to the finite case plus the requirement that $F$ be $\mathfrak{n}-\aleph_{0}$ compact for $\mathfrak{n}=$ card $A$, Theorem 3.3 gives a sufficient condition that such a basis exists (which by Theorem 3.4 can also be necessary) and Corollary 3.6 gives a necessary condition that such a basis exists.

3.7. COROLlaRY. The proper maps form the largest class of closed maps which is closed under arbitrary products.

Proof. If $p$ is in such a class, then by Theorem 3.3 each fiber of $p$ is compact, so $p$ is proper. That products of proper maps are proper follows by 3.3 and 3.4 (and is, of course, well known).

3.8. COROLlaRY. Let $p=\prod_{n \in N} p_{n}$ where each $p_{n}$ is a closed map. If each fiber of each $p_{n}$ is sequential and sequentially compact, then $p$ is closed. 
Proof. A sequentially compact space is countably compact, each finite (indeed, countable) product of sequentially compact spaces is sequentially compact, and the projection along a countably compact space onto a sequential space is closed. Thus 3.3 and 3.4 apply.

3.9. EXAMPLE. For each infinite cardinal $\mathfrak{n}$, there exists an open and closed map $p$ such that $p^{\mathfrak{n}}$ is closed (and open) but for $\mathfrak{m}>\mathfrak{n}, p^{\mathfrak{m}}$ is not closed.

Proof. Let $Y$ be as in Example 1.7, let $Y^{\prime}$ be the disjoint union of two copies of $Y$ and let $p$ be the map on $Y^{\prime}$ which identifies each copy to a point.

4. m-n-compact products. In this section we apply Theorem 1.8 to give conditions under which products will be $\mathfrak{m}-\mathfrak{n}$-compact. Except for treating $\mathfrak{m}-\aleph_{0}$ compactness (and compactness) this method applies only to countable products.

4.1. THeorem. Let $X=\prod_{n} X_{n}$, where each $X_{n}$ is $\mathfrak{m}$-n-compact. If each $X_{n}$ is $<\mathfrak{n}$-discrete and each of its points has a neighborhood base of cardinality at most $\mathfrak{m}$, then $X$ is $\mathfrak{m}$-n-compact.

Proof. Recall that a space $X$ is m-n-compact if and only if $\pi: X \times Y \rightarrow Y$ is closed whenever $Y$ is $<\mathfrak{n}$-discrete and each point of $Y$ has a neighborhood base of cardinality at most $\mathfrak{m}$. Note that if $Y$ is such a space, then so is $\left(\prod_{i=1}^{n-1} X_{i}\right) \times Y$ so the projection $\left(\prod_{i=1}^{n} X_{i}\right) \times Y \rightarrow\left(\prod_{i=1}^{n-1} X_{i}\right) \times Y$ is closed and therefore, by Theorem $1.8, \pi: X \times Y \rightarrow Y$ is closed.

4.2. Corollary. Each countable product of $\mathfrak{n}$-compact, $<\mathfrak{n}$-discrete spaces is $\mathfrak{n}$-compact.

Proof. For each sufficiently large $\mathfrak{n}$, the product is $\mathfrak{m}-\mathfrak{n}$-compact by Theorem 4.2.

Even the case $\mathfrak{n}=\boldsymbol{\aleph}_{0}$ of Corollary 4.2 (that the product of countably many Lindelöf $P$-spaces is Lindelöf) is new and answers affirmatively a question posed (in conversation) by A. W. Hager. By providing a new class of spaces whose countable products are paracompact, it also answers a question posed by E. Michael. Indeed,

4.3. Corollary. Each countable product of completely regular, $\mathfrak{n}$-compact, $<\mathfrak{n}$-discrete spaces is paracompact.

Proof. Let $X=\prod_{n} X_{n}$ be such a product. Since each compact space is paracompact, we may suppose that $\mathfrak{n}$ is greater than $\boldsymbol{N}_{0}$, so each factor is a $P$-space. By complete regularity, each factor has a basis of cozero sets which, being $F_{\sigma}$ 's, are closed as well as open. Thus given any open cover of $X$ we refine and take subcovers (using Corollary 4.2) so as to get a refinement $\mathscr{U}=\left\{U_{\alpha}=\prod_{i=1}^{n(\alpha)} U_{\alpha}^{i} \times \prod_{i>n(\alpha)} X_{i}: \alpha \in A\right\}$ where each $U_{\alpha}^{i}$ is closed and open, $n(\alpha)$ is finite, and card $A<\mathfrak{n}$. For each $n$, let $\mathscr{U}_{n}=\left\{U_{\alpha}: n(\alpha)=n\right\} ;$ well order $\mathscr{U}_{n}$ as $\left\{U_{\lambda}: \lambda \in W\right\}$ and let $\mathscr{U}_{n}^{\prime}=\left\{U_{\lambda} \mid \bigcup_{\delta<\lambda} U_{\delta}: \lambda \in W\right\}$. Since $\prod_{i=1}^{n(\alpha)} X$ is $<\mathfrak{n}$-discrete and card $\left(\mathscr{U}_{n}\right)<\mathfrak{n}$, each member of $\mathscr{U}_{n}^{\prime}$ is open (and closed) and the union of $\mathscr{U}_{n}^{\prime}$ is closed. Thus $\mathscr{U}_{n}^{\prime}$ is locally finite in $X$, and therefore 
$\bigcup_{n} \mathscr{U}_{n}^{\prime}$ is a $\sigma$-locally finite refinement of our original cover. Since $X$ is regular, it follows that $X$ is paracompact.

The restriction, in Theorem 4.1, to countable products was necessitated by the fact that infinite products are not, for $\mathfrak{n}>\boldsymbol{\aleph}_{0}$, $<\mathfrak{n}$-discrete. Of course, for $\mathfrak{n}=\boldsymbol{\aleph}_{0}$ this is no problem since every topological space is $\left\langle\boldsymbol{\aleph}_{0}\right.$-discrete. The other hypothesis, that each point have a neighborhood basis of cardinality at most $\mathfrak{m}$, is of course preserved by $m$-fold products. Thus, by the obvious adaptation of the proof of Theorem 4.1, we have

4.4. ThEOREM. Let $X=\prod_{\alpha \in A} X_{\alpha}$ where each $X_{\alpha}$ is $\mathfrak{n}-\aleph_{0}$-compact and each of its points has a neighborhood basis of cardinality at most $\mathfrak{n}$. If the cardinality of $A$ is less than or equal to the successor of $\mathfrak{n}$, then $X$ is $\mathfrak{n}-\aleph_{0}$-compact.

In the case $\mathfrak{n}=\boldsymbol{\aleph}_{0}$ we can give a somewhat stronger result. To do so, we need

4.5. THEOREM. Each countable product of countably compact sequential spaces is countably compact sequential.

Proof. Let $X=\prod_{n} X_{n}$ be such a product. As in the proof of Theorem 1.6, the product of two countably compact sequential spaces is countably compact, and it is shown in [16] that such a product is sequential. Thus, each finite subproduct of $X$ is sequential and, applying Theorem $1.8, X$ is countably compact. (A more direct proof that $X$ is countably compact is indicated in [23].) It remains to show that $X$ is sequential, so suppose that $S \subseteq X$ contains the limits of each of its convergent sequences; we will show that $S$ is closed.

First suppose that for each $n$ the projection, $\pi_{n}(S)$, of $S$ onto $\prod_{i=1}^{n} X_{i}$ is closed. Choose $x$ in the complement of $S$. If for some $n, \pi_{n}(x)$ is not in $\pi_{n}(S)$, then $\left(\pi_{n}\right)^{-1}\left(\prod_{i=1}^{n} X_{i} \mid \pi_{n}(S)\right)$ is a neighborhood of $x$ which does not meet $S$. On the other hand, if $\pi_{n}(x)$ is in $\pi_{n}(S)$ for each $n$, then choosing $x_{n}$ in $S$ with $\pi_{n}(x)=\pi_{n}\left(x_{n}\right)$, the sequence $\left\{x_{n}\right\}$ converges to $x$, which contradicts our assumption that $S$ contains the limits of its convergent sequences. Thus when each $\pi_{n}(S)$ is closed, $S$ is closed.

Now suppose that for some integer $m, \pi_{m}(S)$ is not closed. Since $\prod_{i=1}^{m} X_{i}$ is sequential, it follows that some sequence $\left\{x_{n}\right\}$ in $\pi_{m}(S)$ converges to a point $x$ in the complement of $\pi_{m}(S)$. Choose $y_{n}$ in $\prod_{i>m} X_{i}$ such that $\left(y_{n}, x_{n}\right)$ is in $S$. Since each $X_{i}$ is (like any countably compact sequential space) sequentially compact, $\prod_{i>m} X_{i}$ is sequentially compact so $\left\{y_{n}\right\}$ has a convergent subsequence $\left\{y_{n_{k}}\right\} \rightarrow y$. But then $\left\{\left(x_{n_{k}} y_{n_{k}}\right)\right\} \rightarrow(x, y)$ so $(x, y)$ is in $S$. Since $x$ is not in $\pi_{n}(S)$, this gives the desired contradiction.

We call a space subsequential if it can be embedded as a subspace of a sequential space. Subsequential spaces form the smallest category containing metric spaces which is closed under the formation of sums, quotients and subspaces. Surprisingly, subsequential spaces are as well behaved under products as are metric spaces:

4.6. THEOREM. Each countable product of subsequential spaces is subsequential. 
Proof. By Theorem 4.5 it suffices to show that each sequential space can be embedded in a countably compact sequential space. We sketch the construction. Starting with a sequential space $X_{0}$, construct $X_{\alpha}$, for each $\alpha \leqq \aleph_{1}$, as follows:

(i) If $\alpha$ is a limit ordinal, let $X_{\alpha}$ be the topological union of $\left\{X_{\beta}: \beta<\alpha\right\}$, i.e., the quotient of the disjoint union induced by the embedding maps $X_{\beta} \rightarrow X_{\delta}$ for $\beta<\delta$.

(ii) If $\alpha$ is not a limit ordinal, form $X_{\alpha}$ from $X_{\alpha-1}$ by adjoining a sequential limit point, $x_{s}$, for each sequence $s \subseteq X_{\alpha-1}$ which has no convergent subsequences. (So in $X_{\alpha}$, the sequence $s$ converges to $x_{s}$.)

Since quotients of sequential spaces are sequential, each $X_{\alpha}$ is sequential, and in particular $X_{\aleph_{1}}$ is sequential. Furthermore, since each sequence in $X_{\aleph_{1}}$ is contained in some $X_{\alpha}$ and therefore has a limit (in $X_{\alpha+1}$ ), $X_{\aleph_{1}}$ is countably compact, and is therefore as desired.

4.7. THEOREM. Each $\boldsymbol{\aleph}_{1}$-fold product of countably compact subsequential spaces is countably compact.

Proof. By Theorems 1.4, 1.8, and 4.6, the projection from such a product onto each of its countable subproducts is closed, so by Theorem 1.2 such a product must be countably compact.

4.8. Corollary (SCARbOROUgh-STONE [27]). Each $\boldsymbol{\aleph}_{1}$-fold product of sequentially compact spaces is countably compact.

Proof. From Theorem 4.7 it follows that each $\aleph_{1}$-fold product of spaces which are continuous images of countably compact subsequential spaces is countably compact. This class includes all sequentially compact spaces (a space is sequentially compact if and only if it is a continuous image of a countably compact sequential space).

Incidentally, the proof of Tychonoff's theorem using Theorem 1.8 is also of some interest. Its use of the axiom of choice differs from that of other proofs in that it restricted to statements about the index set-see [25].

5. Generalizations to products with nonstandard topologies. In this section we extend the results of previous sections to the $\mathfrak{n}$-products defined below. For $\mathfrak{n}$ a regular cardinal, the $\mathfrak{n}$-product is the natural product in the category of $<\mathfrak{n}$ discrete spaces. These products have been studied in [10], [14] and [1].

The n-product $\prod_{\alpha \in A}^{\mathfrak{n}} X_{\alpha}$ of $\left\{X_{\alpha}: \alpha \in A\right\}$ is the set $\prod_{\alpha \in A} X_{\alpha}$ endowed with the smallest topology making continuous all projections $\pi_{B}$ where $B \subseteq A$ has cardinality less than $\mathfrak{n}$. Thus the $\boldsymbol{\aleph}_{0}$-product is the usual product and if $\mathfrak{n}>\operatorname{card}(A)$ the $\mathfrak{n}$ product is the box product. If $\mathfrak{n}$ is regular and each $X$ is $<\mathfrak{n}$-discrete, then clearly $\prod_{\alpha}^{\mathfrak{n}} X_{\alpha}$ is $<\mathfrak{n}$-discrete. If $\mathfrak{n}$ is not regular, i.e. if $\mathfrak{n}$ is the union of fewer than $\mathfrak{n}$ sets each of cardinality less than $\mathfrak{n}$, then $\prod_{\alpha \in \mathfrak{m}}^{\mathfrak{n}} X_{\alpha}$ is not $<\mathfrak{n}$-discrete for $\mathfrak{m} \geqq \mathfrak{n}$. Of course, for such $\mathfrak{n}$ each $<\mathfrak{n}$-discrete space is $\mathfrak{n}$-discrete and hence $<\mathfrak{n}^{\prime}$-discrete for $\mathfrak{n}^{\prime}$ the successor of $\mathfrak{n}$, and $\mathfrak{n}^{\prime}$ is regular, so the $\mathfrak{n}^{\prime}$-product is the natural product for the category of $<\mathfrak{n}$-discrete spaces. The proofs of the previous sections adapt easily, except where noted, to establish the following results. 
5.1. TheOREM. Let $\mathfrak{n}$ be regular and let $X=\prod_{\alpha \in \mathfrak{m}}^{\mathfrak{n}} X_{\alpha}$ where $\mathfrak{m}$ is infinite and each $X_{\alpha}$ is completely regular.

(i) If $\mathfrak{m} \geqq \mathfrak{n}$, then each projection on $X$ is $z$-closed if and only if $X$ is pseudo-ncompact and $<\mathfrak{n}$-discrete.

(ii) If $\mathfrak{m}<\mathfrak{n}, B=\left\{\alpha \in \mathfrak{m}: X_{\alpha}\right.$ is discrete $\}$, and $\mathfrak{n}^{\prime}=$ card $\left(\prod_{\alpha \in B} X_{\alpha}\right)$, then each projection on $X$ is z-closed if and only if for some cardinal $\mathfrak{n}^{\prime \prime}>\mathfrak{n}^{\prime}$ the space $\prod_{\alpha \in \mathfrak{m} \backslash B} X_{\alpha}$ is pseudo-n"-compact and $<\mathfrak{n}^{\prime \prime}$-discrete. (Note: $\mathfrak{n}^{\prime \prime}$ will have to be larger than $2^{\operatorname{card}(\mathfrak{m} \backslash B)}$.)

5.2. Theorem. Let $\mathfrak{n}$ be regular, and let $X=\prod_{\alpha \in \mathfrak{m}}^{\mathfrak{n}} X_{\alpha}$ where $\mathfrak{m} \geqq \mathfrak{n}$ and each $X_{\alpha}$ is $<\mathfrak{n}$-discrete. Then each projection on $X$ is closed if and only if $X$ is $\mathfrak{m}$-n-compact and each box product of fewer than $\mathfrak{n}$ of the $X_{\alpha}$ has each projection closed.

5.3. TheOREM. Let $X=\prod_{\alpha \in \mathfrak{m}}^{\mathfrak{n}} X_{\alpha}$ where $\mathfrak{m} \geqq \mathfrak{n}$, each $X_{\alpha}$ is $<\mathfrak{n}$-discrete and each box product of fewer than $\mathfrak{n}$ of the $X_{\alpha}$ is normal $T_{1}$.

(i) If $X$ is $\mathfrak{m}$-n-compact, then $X$ is normal.

(ii) If $\mathfrak{m}>\mathfrak{n}$, if $\mathfrak{n}$ is regular and for some $\mathfrak{n}^{\prime}<\mathfrak{n}$ one has $2^{\mathfrak{n}^{\prime}} \geqq \mathfrak{n}$, then $X$ is not normal (or $\mathfrak{m}$-n-compact).

(iii) If $X$ is normal, if $\mathfrak{n}$ is regular, and for each $\mathfrak{n}^{\prime}<\mathfrak{n}$ one has $2^{\mathfrak{n}^{\prime}}<\mathfrak{n}$, then some product of all but $\mathfrak{n}$ of the factors of $X$ is $\mathfrak{m}$-n-compact.

Proof. Part (i) follows by the obvious adaptation of the proof of the corresponding part of Theorem 2.1 (Lemma 1.5 carries over easily).

(ii) It is shown in [1] that for $\mathfrak{n}$ regular the $\mathfrak{n}$-product of more than $\mathfrak{n}$ discrete spaces each of cardinality $\mathfrak{n}$ is not normal. Since each $\mathfrak{n}^{\prime}$-fold box product (of nontrivial $T_{1}$ spaces) contains a closed discrete subset of cardinality $2^{\mathfrak{n}^{\prime}}$, (ii) follows.

(iii) By the result from [1] quoted above, all but $\mathfrak{n}$ of the factors must be pseudo$\mathfrak{n}$-compact. But then, adapting the proofs of [24], some $\mathfrak{n}$-product of all but $\mathfrak{n}$ of the factors must be pseudo-n-compact. The projections on this product are $z$-closed by Theorem 5.1, hence (by normality) closed, so by Theorem 5.2 the product is m-n-compact.

5.4. TheOREM. Let $\mathfrak{n}$ be regular, and let $p$ be the $\mathfrak{n}$-product of closed maps $\left\{p_{\alpha}: \alpha \in \mathfrak{m}\right\}$ where $\mathfrak{m} \geqq \mathfrak{n}$ and each fiber of each $p_{\alpha}$ is $<\mathfrak{n}$-discrete. The map $p$ is closed if and only if each fiber of $p$ is $\mathfrak{m}-\mathfrak{n}$-compact and each box product of fewer than $\mathfrak{n}$ of the $p_{\alpha}$ is closed.

5.5. Theorem. Let $\mathfrak{n}$ be regular, let $X=\prod_{\alpha \in \mathfrak{m}}^{\mathfrak{n}} X_{\alpha}$ and let $Y$ be any space. Suppose that for each box product $X^{\prime}$ of fewer than $\mathfrak{n}$ of the $X_{\alpha}$, the projection $X^{\prime} \times Y \rightarrow Y$ is closed. If for each $\alpha_{0}$ in $\mathfrak{m}$ the projection $X_{\alpha_{0}} \times\left(\prod_{\alpha<\alpha_{0}}^{\mathfrak{n}} X_{\alpha}\right) \times Y \rightarrow \prod_{\alpha<\alpha_{0}}^{\mathfrak{n}} X_{\alpha} \times Y$ is closed, then the projection from $X \times Y$ to $Y$ is closed.

5.6. TheOrem. Let $\mathfrak{n}$ be regular, and let $X=\prod_{\alpha \in \mathfrak{n}}^{\mathfrak{n}} X_{\alpha}$ where each $X_{\alpha}$ is $<\mathfrak{n}$ discrete and each box product of fewer than $\mathfrak{n}$ of the $X_{\alpha}$ is $\mathfrak{m}$-n-compact. Let $\mathfrak{m}^{\prime}$ be a cardinal such that whenever $\mathfrak{n}^{\prime}$ is less than $\mathfrak{n},\left(\mathfrak{m}^{\prime}\right)^{\mathfrak{n}^{\prime}}$ is less than or equal to $\mathfrak{m}$. If 
each point of each $X_{\alpha}$ has a neighborhood base of cardinality at most $\mathfrak{m}^{\prime}$, then $X$ is $\mathfrak{m}$-n-compact.

Note that the requirement that box products of fewer than $\mathfrak{n}$ factors be $\mathfrak{m}-\mathfrak{n}$ compact forces $\mathfrak{n}$ to be $\boldsymbol{\aleph}_{0}$ or strongly inaccessible, so any cardinal less than $\mathfrak{n}$ satisfies the hypothesis on $\mathfrak{m}^{\prime}$. If $\mathfrak{n}$ is strongly inaccessible and each $X_{\alpha}$ has a base of cardinality less than $\mathfrak{n}$, then the theorem applies to show that $\prod_{\alpha \in \mathfrak{n}}^{\mathfrak{n}} X_{\alpha}$ is $\mathfrak{n}$ compact. Of course, strongly inaccessible cardinals need not be assumed to exist.

\section{REFERENCES}

1. C. J. R. Borges, On a counter-example of A. H. Stone, Quart. J. Math. Oxford Ser. (2) 20 (1969), 91-95. MR 40 \#6489.

2. W. W. Comfort, A nonpseudocompact product space whose finite subproducts are pseudocompact, Math. Ann. 170 (1967), 41-44. MR 35 \#965.

3. W. W. Comfort and A. W. Hager, The projection mapping and other continuous functions on a product space (to appear).

4. - Estimates for the number of real-valued continuous functions, Trans. Amer. Math. Soc. 150 (1970), 619-632.

5. I. Fleischer and S. P. Franklin, On compactness and projections, Internationale Spezialtagung für Erweiterungstheorie topologischer Strukturen und deren Anwendungen, Berlin, 1967.

6. Z. Frolik, The topological product of countably compact spaces, Czechoslovak Math. J. 10 (85) (1960), 329-338. MR 22 \#8480.

7. - On two problems of W. W. Comfort, Comment. Math. Univ. Carolinae 8 (1967), 139-144. MR 35 \#966.

8. — Sums of ultrafilters, Bull. Amer. Math. Soc. 73 (1967), 87-91. MR 34 \#3525.

9. I. Glicksberg, Stone-Čech compactifications of products, Trans. Amer. Math. Soc. 90 (1959), 369-382. MR 21 \#4405.

10. G. Gould, Locally unbounded topological fields and box topologies on products of vector spaces, J. London Math. Soc. 36 (1961), 273-281. MR 24 \#A413.

11. S. L. Gulden, On m-sequential spaces, Notices Amer. Math. Soc. 16 (1969), 293. Abstract \#663-706.

12. A. W. Hager, Projections of zero-sets (and the fine uniformity on a product), Trans. Amer. Math. Soc. 140 (1969), 87-94. MR 39 \#3448.

13. J. L. Kelley, General topology, Van Nostrand, Princeton, N. J., 1955. MR 16, 1136.

14. C. J. Knight, Box topologies, Quart. J. Math. Oxford Ser. (2) 15 (1964), 41-54. MR 28 \#3398.

15. E. Michael, The product of a normal space and a metric space need not be normal, Bull. Amer. Math. Soc. 69 (1963), 375-376. MR 27 \#2956.

16. - Local compactness and Cartesian products of quotient maps and k-spaces, Ann. Inst. Fourier (Grenoble) 18 (1968), fasc. 2, 281-286. MR 39 \#6256.

17. K. Morita, Paracompactness and product spaces, Fund. Math. 50 (1961/62), 223-236. MR 24 \#A2365.

18. S. Mrowka, On function spaces, Fund. Math. 45 (1957), 273-282.

19. N. Noble, Products with closed projections, Trans. Amer. Math. Soc. 140 (1969), 381-391. MR 40 \#3500.

20. _- Ascoli theorems and the exponential map, Trans. Amer. Math. Soc. 143 (1969), 393-411. MR 40 \#1978.

21. —-, A note on z-closed projections, Proc. Amer. Math. Soc. 23 (1969), 73-76. MR 39 \#7575. 
22. N. Noble, Products of quotient maps and spaces with weak topologies (to appear).

23. - Countably compact and pseudo-compact products, Czechoslovak Math. J. 19 (94) (1969), 390-397. MR 40 \#1968.

24. - A generalization of a theorem of A. H. Stone, Arch. Math. (Basel) 18 (1967), 394-395. MR 36 \#5883.

25. —, A nice proof of Tychonoff's theorem (to appear).

26. C. T. Scarborough, Closed graphs and closed projections, Proc. Amer. Math. Soc. 20 (1969), 465-470. MR 40 \#3514.

27. C. T. Scarborough and A. H. Stone, Products of nearly compact spaces, Trans. Amer. Math. Soc. 124 (1966), 131-147. MR 34 \#3528.

28. R. M. Stephenson, Jr., Pseudocompact spaces, Trans. Amer. Math. Soc. 134 (1968), 437-448. MR 38 \#674.

29. — Product spaces for which the Stone-Weierstrass theorem holds, Proc. Amer. Math. Soc. 21 (1969), 284-288. MR 40 \#3499.

30. H. Tamano, A note on the pseudo-compactness of the product of two spaces, Mem. Coll. Sci. Univ. Kyoto Ser. A Math. 33 (1960/61), 225-230. MR 22 \#11369.

31. J. W. Tukey, Convergence and uniformity in topology, Ann. of Math. Studies, no. 2, Princeton Univ. Press, Princeton, N. J., 1940. MR 2, 67.

UNIVERSITY OF WASHINGTON,

Seattle, Washington 98105 